TITLE:

\title{
Sfermion mass degeneracy, superconformal dynamics, and supersymmetric grand unified theories
}

\section{$\operatorname{AUTHOR}(\mathrm{S}):$}

Kobayashi, T; Nakano, H; Noguchi, T; Terao, H

\section{CITATION:}

Kobayashi, T ...[et al]. Sfermion mass degeneracy, superconformal dynamics, and supersymmetric grand unified theories. PHYSICAL REVIEW D 2002, 66(9): 095011.

\section{ISSUE DATE:}

2002-11-01

URL:

http://hdl.handle.net/2433/50536

RIGHT:

Copyright 2002 American Physical Society 


\title{
Sfermion mass degeneracy, superconformal dynamics, and supersymmetric grand unified theories
}

\author{
Tatsuo Kobayashi, ${ }^{1, *}$ Hiroaki Nakano, ${ }^{2, \dagger}$ Tatsuya Noguchi, ${ }^{1, \hbar}$ and Haruhiko Terao ${ }^{3, \S}$ \\ ${ }^{1}$ Department of Physics, Kyoto University, Kyoto 606-8502, Japan \\ ${ }^{2}$ Department of Physics, Niigata University, Niigata 950-2181, Japan \\ ${ }^{3}$ Institute for Theoretical Physics, Kanazawa University, Kanazawa 920-1192, Japan
}

(Received 11 February 2002; published 25 November 2002)

\begin{abstract}
We discuss issues in a scenario where hierarchical Yukawa couplings are generated through the strong dynamics of superconformal field theories (SCFTs). Independently of the mediation mechanism of supersymmetry breaking, the infrared convergence property of SCFTs can provide an interesting solution to the supersymmetric flavor problem; sfermion masses are suppressed around the decoupling scale of SCFTs and eventually become degenerate to some degree, thanks to family-independent radiative corrections governed by the gaugino masses of the minimal supersymmetric standard model (MSSM). We discuss under what conditions the degeneracy of the sfermion mass can be estimated in a simple manner. We also discuss the constraints from lepton flavor violations. We then explicitly study sfermion mass degeneracy within the framework of grand unified theories coupled to SCFTs. It is found that the degeneracy for right-handed sleptons becomes worse in the conventional SU(5) model than in the MSSM. On the other hand, in the flipped $\mathrm{SU}(5) \times \mathrm{U}(1)$ model, each right-handed lepton is still an $\mathrm{SU}(5)$ singlet, whereas the $B$-ino mass $M_{1}$ is determined by two independent gaugino masses of $S U(5) \times U(1)$. These two properties enable us to have an improved degeneracy for the right-handed sleptons. We also speculate on how further improvement can be obtained in the SCFT approach.

DOI: 10.1103/PhysRevD.66.095011

PACS number(s): 11.30.Pb, 11.10.Hi, 12.10.Dm, 12.60.Jv
\end{abstract}

\section{INTRODUCTION}

Understanding the origin of hierarchical fermion masses and mixing angles is one of the most important issues in particle physics. The Froggatt-Nielsen (FN) mechanism is a famous mechanism for realizing hierarchical Yukawa couplings $[1,2]$. Recently, new ideas related to extra dimensions have also been discussed.

In models with softly broken supersymmetry (SUSY), a mechanism that generates the hierarchical structure of Yukawa couplings generally affects the sfermion sector; one would have a characteristic pattern of sfermion masses and SUSY-breaking trilinear couplings. For example, the FN mechanism with an extra $\mathrm{U}(1)$ gauge symmetry leads to the so-called $D$-term contributions to soft scalar masses, which are proportional to the charges under the broken $\mathrm{U}(1)$ symmetry. [See Ref. [3] for $D$-term contributions through grand unified theory (GUT) breaking and Ref. [4] for anomalous U(1) breaking.] Such a pattern could be tested if superpartners are discovered and sfermion masses as well as trilinear scalar couplings are measured in future experiments. Even at present, soft SUSY-breaking parameters are constrained rather severely from the experimental bounds on flavor changing neutral current (FCNC) processes as well as $C P$ violation. This is the SUSY flavor problem. For instance, the flavor-dependent $D$-term contributions are generically dangerous. In general, the SUSY flavor problem can be solved if one of the following three conditions is realized at least for the first two families: (1) diagonal and degenerate sfermion

\footnotetext{
*Electronic address: kobayash@gauge.scphys.kyoto-u.ac.jp

${ }^{\dagger}$ Electronic address: nakano@muse.sc.niigata-u.ac.jp

*Electronic address: noguchi@gauge.scphys.kyoto-u.ac.jp

${ }^{\S}$ Electronic address: terao@hep.s.kanazawa-u.ac.jp
}

masses; (2) decoupling of heavy sfermions; and (3) alignment between the fermion and sfermion bases. Much effort has been devoted to realizing the first solution by seeking a flavor-blind mediation mechanism of SUSY breaking.

Nelson and Strassler [5] have recently proposed an interesting mechanism for realizing hierarchical Yukawa couplings. The setup is the minimal supersymmetric standard model (MSSM), or its extension, coupled to the superconformal (SC) sector. The SC sector is strongly coupled and assumed to have an infrared (IR) fixed point [6,7]. The first and second families of quarks and leptons gain large and positive anomalous dimensions through the SC dynamics. Then their Yukawa couplings to electroweak Higgs fields are suppressed hierarchically at the scale $M_{C}$ where the SC sector is assumed to decouple from the MSSM sector.

The SC fixed point has more interesting consequences. When pure superconformal field theory (SCFT) is perturbed by soft SUSY-breaking terms, general argument shows that this perturbation is exponentially suppressed toward the SC fixed point [8-11]. Specifically, one expects that a sfermion mass is suppressed at the decoupling scale $M_{C}$ and eventually receives radiative corrections governed by the MSSM gaugino masses, which are flavor blind. Hence, we would have a degenerate mass spectrum of sfermions like the "noscale" model (at least for the first two families). This possibility has already been mentioned in Ref. [5]. In this scenario, the soft scalar masses are controlled by the flavor mechanism that generates hierarchical Yukawa couplings, no matter how SUSY breaking is mediated and no matter what the initial conditions of the soft SUSY-breaking terms are. This approach, which we shall pursue in this paper, is quite opposite to the usual scenario in which the degenerate soft scalar masses are supposed to be derived by a flavor-blind mediation of SUSY breaking.

When the pure SCFT is perturbed by standard model 
(SM) gauge interactions and the MSSM gaugino masses, each sfermion mass is not completely suppressed, but converges on a flavor-dependent value [10,11]. The convergent value is one-loop suppressed since the SM gauge couplings are perturbatively small. It is then plausible that sfermion masses at the weak scale may be calculated solely in terms of the SM gauge coupling constants and gaugino masses. In fact, under some assumptions, we can estimate the degeneracy factor $\Delta_{\tilde{f}}$ of sfermion masses up to a single modeldependent parameter $\Gamma_{i}$ [10]. It was found that for $M_{C}$ $>10^{10} \mathrm{GeV}$, the degeneracy factor is $0.005-0.01$ for squarks and $0.05-0.1$ for sleptons. In particular, the right-handed sleptons are not well degenerate in mass. Note that these degeneracy factors are evaluated in the sfermion basis.

In this paper, we first examine the assumptions that are implicit in the estimation of sfermion mass degeneracy in the present SCFT approach. We also discuss to what extent the degeneracy is required in the Nelson-Strassler scenario. With this aim, we take into account the fact that FCNC processes, when correctly evaluated in the fermion basis, have additional suppression since the Nelson-Strassler (NS) mechanism leads to hierarchical Yukawa matrices.

It is natural to extend the analysis to grand unified theories and to examine how much degeneracy of sfermion masses is achieved by coupling to SCFTs. That is our second purpose; we explicitly study sfermion mass degeneracy within the framework of GUTs coupled to SC sectors. We take the $\mathrm{SU}(5)$ and the flipped $\mathrm{SU}(5) \times \mathrm{U}(1)$ as a prototype of GUT models. We shall show how much degeneracy of sfermion masses is expected in each case. It turns out that a simple extention of the MSSM to SU(5) makes the degeneracy of the right-handed sleptons worse, because each righthanded lepton is embedded into a higher-dimensional representation. The situation is different in the flipped SU(5) $\times \mathrm{U}(1)$ case, since the right-handed leptons remain $\mathrm{SU}(5)$ singlets [12], and the $B$-ino mass $M_{1}$ is determined by a combination of two independent gaugino masses of $\mathrm{SU}(5)$ $\times \mathrm{U}(1)$.

This paper is organized as follows. After a brief review on the Nelson-Strassler mechanism in Sec. II A, we outline in Sec. II B the results of Ref. [10] on the degeneracy of sfermion masses in the sfermion basis within the framework of the MSSM coupled to SC sectors. We also discuss under what conditions the degeneracy factor can be estimated in a simple manner. The FCNC constraints are examined in Sec. II C by taking into account Yukawa-diagonalizing matrices. In Secs. III and IV, we extend the model to GUTs and investigate generic features. Specifically we argue that the degeneracy of the right-handed sleptons becomes worse in SU(5) models than in the MSSM. We then show how the degeneracy for the right-handed sleptons can be improved in the flipped $\mathrm{SU}(5) \times \mathrm{U}(1)$ models. In Sec. V, we briefly discuss various sorts of threshold effects, which might affect the previous results. This in particular includes $D$-term contributions on sfermion masses in the flipped $\mathrm{SU}(5) \times \mathrm{U}(1)$ models. Section VI is devoted to conclusions and discussion.

We note that Luty and Sundrum [13] discuss a scenario of "conformal sequestering," which is another interesting approach to the SUSY flavor problem based on four- dimensional SCFTs. Although closely related to the present SCFT approach, it is slightly different in that the SCFT couples to messenger fields of SUSY breaking, not to quarks and leptons.

\section{MSSM COUPLED TO SC SECTOR}

\section{A. Nelson-Strassler mechanism for Yukawa hierarchy}

Here we give a brief review on the Nelson-Strassler mechanism that generates the hierarchical structure of Yukawa couplings [5]. We assume two sectors: One is the $\mathrm{SM}$ sector and the other is the SC sector. The SM sector has the gauge group $\mathrm{SU}(3) \times \mathrm{SU}(2) \times \mathrm{U}(1)_{Y}$, or its extension, and contains three families of quarks and leptons $\psi_{i}(i$ $=1,2,3)$ as well as Higgs fields $H$. The SC sector has a gauge group $G_{\mathrm{SC}}$ and matter fields $\Phi_{r}$. The fields $\psi_{i}$ and $H$ are taken to be $G_{\mathrm{SC}}$ singlets. The following superpotential is assumed:

$$
W=y_{i j} \psi_{i} \psi_{j} H+\lambda_{i r s} \psi_{i} \Phi_{r} \Phi_{s}+\cdots,
$$

where the first term describes the ordinary Yukawa couplings in the SM sector and the ellipsis denotes terms including only $\Phi_{r}$. The second term represents the couplings of quarks and leptons $\psi_{i}$ to the SC sector, which we refer to as messenger couplings. For the messenger coupling $\lambda_{i r s}$ to be allowd by gauge invariance, either of $\Phi_{r}$ or $\Phi_{s}$ should belong to a nontrivial representation under the SM gauge group.

With sufficiently many matter fields, the $G_{\mathrm{SC}}$ gauge theory resides in a "conformal window;" the SC gauge coupling $g^{\prime}$ has an IR fixed point [6,7]. Suppose that the messenger couplings $\lambda_{i r s}$ as well as $g^{\prime}$ approach IR fixed points. At this new fixed point, the field $\psi_{i}$ gains a large anomalous dimension $\gamma_{i}^{*}=\mathcal{O}(0.1-1)$ through superconformal dynamics. As a result, the Yukawa couplings in the SM sector obey a power law and behave roughly like

$$
y_{i j}\left(M_{C}\right) \approx y_{i j}\left(M_{0}\right)\left(\frac{M_{C}}{M_{0}}\right)^{\gamma_{i}^{*}+\gamma_{j}^{*}} .
$$

Here $M_{0}$ is the cutoff scale, at which $y_{i j}\left(M_{0}\right)=\mathcal{O}(1)$ is expected. Thus the hierarchical structure of Yukawa couplings can be generated by family-dependent anomalous dimensions $\gamma_{i}^{*}$. The resultant Yukawa matrices are similar to the ones obtained by the FN mechanism; a large anomalous dimension in the NS mechanism corresponds to a U(1) charge in the FN mechanism. As stressed in Ref. [5], the unitarity of the SCFT guarantees that the anomalous dimensions $\gamma_{i}^{*}$ at the SC fixed point are always non-negative, whereas the nonnegativity of $\mathrm{U}(1)$ charges is just the assumption in the conventional FN mechanism.

Since the top Yukawa coupling should not be suppressed, the top quark as well as the up-type Higgs field must not couple to the SC sector. Although the bottom quark and tau lepton as well as the down-type Higgs could couple, we will mainly consider, in what follows, models in which only the first two families couple to the SC sector. 


\section{B. Degeneracy of sfermion masses}

Next we outline, following Ref. [10], how convergent values of the soft scalar masses and sfermion mass degeneracy can be estimated. See also Ref. [14] for a review. We also discuss some subtleties of this estimation.

The suppression of sfermion masses in the SCFT approach follows from a general property of the renormalization group equations (RGEs) for soft SUSY-breaking parameters $[14,15]$. Let us concentrate for a moment on the diagonal elements $m_{i}^{2}$ of a sfermion mass-squared matrix [in a flavor basis in which the fermion Yukawa matrix takes the form (2)]. Near an IR attractive fixed point of pure SCFT, the RGE for $m_{i}^{2}$ in the SM sector as well as the SC sector takes the form

$$
\mu \frac{d m_{i}^{2}}{d \mu}=\mathcal{M}_{i j} m_{j}^{2}
$$

where the coefficient matrix $\mathcal{M}_{i j}$ encodes the full effects of the SC dynamics, and can be calculated by use of the "Grassmanian expansion method" if the anomalous dimension $\gamma_{i}$ is known as a function of the coupling constants $g^{\prime}$ and $\lambda_{i r s}$. This matrix is positive definite (non-negative) since the fixed point is IR attractive. It follows that certain combinations of $m_{i}^{2}$ are exponentially suppressed. Moreover, each $m_{i}^{2}$ is suppressed if its anomalous dimension $\gamma_{i}^{*}$ is uniquely determined $[5,10]$ by the fixed point equations $\beta_{g^{\prime}}=\beta_{\lambda}=0$. We assume that in each model considered below there exists a proper set of couplings that satisfies this condition.

When we switch on the gauge couplings $\alpha_{a}=g_{a}^{2} / 8 \pi^{2}$ and gaugino masses $M_{a}$ in the SM sector $(a=1,2,3)$, the RGE (3) gets modified, at leading order, into

$$
\mu \frac{d m_{i}^{2}}{d \mu}=\mathcal{M}_{i j} m_{j}^{2}-\sum_{a=1,2,3} C_{i a} \alpha_{a} M_{a}^{2}
$$

where $C_{i a}=4 C_{2}\left(R_{i a}\right)$ with the quadratic Casimir coefficient $C_{2}\left(R_{i a}\right)$ of the $R_{i a}$ representation, and we have neglected for simplicity a possible contribution from the $\mathrm{U}(1)_{Y}$ FayetIliopoulos term $\mathcal{S} \equiv \operatorname{Tr}\left(Y m_{i}^{2}\right)$. Equation (4) implies that irrespective of initial values each sfermion mass converges on the one-loop suppressed value of order $\alpha_{a} M_{a}^{2}$. This value, which we will call the "convergent value," generally depends on the detailed structure of the SC sector, since the above RGEs are coupled equations for the soft scalar masses in the SC sector as well as those of squarks and sleptons.

We are interested in the convergent values of squark and slepton masses at the scale $M_{C}$ where the SC sector decouples. Let $m_{\tilde{f} i}$ denote the soft scalar mass of the $i$ th family of squarks or sleptons $\widetilde{f}$. Then the convergent value of $m_{\tilde{f} i}^{2}$ at $M_{C}$ can be written in the form [10]

$$
m_{\tilde{f} i}^{2} \rightarrow \frac{1}{\Gamma_{\tilde{f} i}} \sum_{a=1,2,3} C_{f a} \alpha_{a}\left(M_{C}\right) M_{a}^{2}\left(M_{C}\right) .
$$

TABLE I. Group-theoretical factors for $\Delta_{\bar{f}}$. Our normalization convention for the $\mathrm{U}(1)$ gauge coupling is the $\mathrm{SU}(5)$-motivated one, $\alpha_{1} \equiv(5 / 3) \alpha_{Y}$.

\begin{tabular}{lccccc}
\hline \hline$f$ & $a_{f 3}$ & $a_{f 2}$ & $a_{f 1}$ & $\begin{array}{c}\mathrm{SU}(5) \\
\text { representation }\end{array}$ & $C_{f 5}$ \\
\hline$Q$ & $-8 / 9$ & $3 / 2$ & $1 / 198$ & $\mathbf{1 0}$ & $72 / 5$ \\
$u$ & $-8 / 9$ & 0 & $8 / 99$ & $\mathbf{1 0}$ & $72 / 5$ \\
$d$ & $-8 / 9$ & 0 & $2 / 99$ & $\overline{\mathbf{5}}$ & $48 / 5$ \\
$L$ & 0 & $3 / 2$ & $1 / 22$ & $\overline{\mathbf{5}}$ & $48 / 5$ \\
$e$ & 0 & 0 & $2 / 11$ & $\overline{\mathbf{1 0}}$ & $72 / 5$ \\
\hline
\end{tabular}

In this expression, $C_{f a}$ is the Casimir factor for $f=Q, \mathrm{u}, \mathrm{d}$, $\mathrm{L}, \mathrm{e}$, and the prefactor $\Gamma_{\tilde{f} i}^{-1}$ summarizes the structure of the SC sector. Typically, we find $\Gamma_{i} \sim \gamma_{i}^{*}=\mathcal{O}(0.1-1)$ for squarks and sleptons (although the same is not always true for sfermions in the SC sector). If $\gamma_{i}^{*}$ and $\gamma_{j}^{*}$ are different from each other, the factors $\Gamma_{\tilde{f} i}$ and $\Gamma_{\tilde{f} j}$ are also different. Only the prefactor $\Gamma_{\tilde{f} i}^{-1}$ has flavor dependency. Consequently, the difference between the first and second families is given by

$$
\begin{gathered}
m_{\tilde{f} 2}^{2}\left(M_{C}\right)-m_{\tilde{f} 1}^{2}\left(M_{C}\right) \\
=\left(\frac{1}{\Gamma_{\tilde{f} 2}}-\frac{1}{\Gamma_{\tilde{f} 1}}\right) \sum_{a=1,2,3} C_{f a} \alpha_{a}\left(M_{C}\right) M_{a}^{2}\left(M_{C}\right),
\end{gathered}
$$

which is also one-loop suppressed compared with $M_{a}^{2}\left(M_{C}\right)$.

We have considered only the diagonal elements of the sfermion mass-squared matrix. However, as was shown in Ref. [11] for pure SCFT, off-diagonal elements are also exponentially suppressed. Even when we switch on the SM effects, the RGEs for off-diagonal elements, unlike Eq. (4), contain no contribution from the MSSM gaugino masses at the leading order. Thus the off-diagonal elements converge on sufficiently small values, which we can safely neglect.

In Ref. [10], sfermion mass degeneracy was examined by assuming the MSSM field content below $M_{C}$. Here let us recall some results for later convenience. The sfermion mass receives the radiative correction $\Delta m_{\tilde{f} i}^{2}$ between $M_{C}$ and $M_{Z}$, which is evaluated to be

$$
\begin{gathered}
\Delta m_{\tilde{f} i}^{2}\left(M_{C} \rightarrow M_{Z}\right)=\sum_{a=1,2,3} a_{f a} I_{a}\left(M_{Z}, M_{C}\right) M_{a}^{2}\left(M_{C}\right), \\
I_{a}\left(M_{Z}, M_{C}\right) \equiv 1-\frac{\alpha_{a}^{2}\left(M_{Z}\right)}{\alpha_{a}^{2}\left(M_{C}\right)}, \quad a_{f a} \equiv \frac{C_{f a}}{2 b_{a}} .
\end{gathered}
$$

Here $b_{a}$ are the MSSM gauge beta-function coefficients, and the factors $a_{f a}(a=1,2,3)$ are shown in the second, third, and fourth columns of Table I for each matter field. These radiative corrections are much larger than the convergent value $m_{\tilde{f} i}^{2}\left(M_{C}\right)$. It follows that 


$$
\left.\frac{m_{\tilde{f} 2}^{2}-m_{\tilde{f} 1}^{2}}{m_{\tilde{f} 2}^{2}+m_{\tilde{f} 1}^{2}}\right|_{M_{Z}}=\frac{1}{2}\left(\frac{1}{\Gamma_{\tilde{f} 2}}-\frac{1}{\Gamma_{\tilde{f} 1}}\right) \Delta_{\tilde{f}},
$$

where we define the degeneracy factor $\Delta_{\tilde{f}}$ by

$$
\Delta_{\tilde{f}}=\frac{\sum_{a} C_{\tilde{f} a} \alpha_{a}\left(M_{C}\right) M_{a}^{2}\left(M_{C}\right)}{\sum_{a} a_{f a} I_{a}\left(M_{Z}, M_{C}\right) M_{a}^{2}\left(M_{C}\right)} .
$$

The factor $\Delta_{f}$ serves as an estimate of how much degeneracy of sfermion masses is achieved in the present framework. Moreover, it is a calculable quantity independently of the detailed structure of the SC sector, especially when the SM gaugino masses satisfy the "GUT" relation $M_{a} / \alpha_{a}=$ const for $a=1,2,3$. For example, taking $M_{C}=10^{16} \mathrm{GeV}$ gives [10]

$$
\begin{aligned}
& \Delta_{\tilde{Q}}=8 \times 10^{-3}, \quad \Delta_{\tilde{u}}=\Delta_{\tilde{d}}=6 \times 10^{-3}, \\
& \Delta_{\tilde{L}}=5 \times 10^{-2}, \quad \Delta_{\tilde{e}}=1 \times 10^{-1} .
\end{aligned}
$$

Unfortunately, the degeneracy factor $\Delta_{\tilde{e}}$ for the right-handed slepton is rather large. The primary reason is that the radiative correction to the right-handed slepton mass, which involves only $M_{1}$, is smaller than the others. ${ }^{1}$ However, this result does not necessarily imply that the present SCFT approach to the Yukawa hierarchy leads to significant lepton flavor violation, because the actual size of lepton flavorviolating processes depends on the explicit form of the lepton Yukawa matrix, as we discuss below.

Some remarks are to be added here. The convergent value (5) is determined by the MSSM one-loop term in the RGE (4). In general, higher-loop terms like $\left(\lambda_{*}^{2} / 8 \pi^{2}\right)^{n} \times \alpha_{a} M_{a}^{2}$ are potentially large because the fixed point value of the messenger coupling $\lambda_{\text {irs }}$ in Eq. (1) is not necessarily small. In particular, the slepton mass $m_{\tilde{e}}^{2}\left(M_{C}\right)$ will be affected by a correction of order $\alpha_{3} M_{3}^{2}$ if the right-handed lepton $e_{\mathrm{R}}^{c}$ couples to colored SC fields through the messenger interactions. Nevertheless, the presence or absence of such corrections depends on the structure of the SC sector; for instance, there is no two-loop term of $\lambda_{e}^{2} \alpha_{3} M_{3}^{2}$ if both of $\Phi_{r}$ and $\Phi_{s}$ are $\mathrm{SU}(3)$ singlets in the messenger interaction $e_{\mathrm{R}}^{c} \Phi_{r} \Phi_{s}$. In the following analysis, we will assume that such higher-loop terms are negligible or at most comparable to the one-loop

\footnotetext{
${ }^{1}$ The degeneracy factor $\Delta_{\tilde{e}}$ for the right-handed sleptons can be somewhat reduced [10] if there is a sizable contribution from the $\mathrm{U}(1)_{Y}$ Fayet-Iliopoulos term $\mathcal{S}$ (with a suitable sign). Note that we generally expect $\mathcal{S} \neq 0$ since soft scalar masses of the third family and Higgs fields are not constrained by SC dynamics.
}

term. ${ }^{2}$ Another remark is that there might arise large threshold effects when the strongly coupled SC sector decouples. In expression (6), we have implicitly assumed that these are negligible or flavor independent. We shall comment on such effects in Sec. V.

In passing, we note the sparticle mass spectrum at the weak scale. As far as the first two families of sfermions are concerned, the convergent values at $M_{C}$ are quite small, $m_{\tilde{f} i}^{2} \approx 0$, and the mass spectrum is similar to that in the "noscale" scenario, provided that there is no large correction at the SC threshold. For $M_{C}=10^{16} \mathrm{GeV}$, we have

$$
\left(m_{\tilde{Q}}, m_{\tilde{L}}, m_{\tilde{e}}\right)=(0.91,0.25,0.13) M_{3} .
$$

The other SU(2)-singlet squarks have masses similar to $m_{\tilde{Q}}$. Again we have taken $\mathcal{S}=0$ for simplicity although nonzero $\mathcal{S}$ is helpful to avoid the charged lightest superparticle (LSP) [10]. On the other hand, the third family, in particular the top quark, as well as the Higgs fields do not couple to the SC sector. In general, their soft scalar masses depend on initial values as well as the details of the RG running of gauge and Yukawa couplings. Hence we restrict ourselves to the first and second families of sfermion masses in the following analysis.

\section{Mixing angles and FCNC constraints}

The factor $\Delta_{\tilde{f}}$ represents a simple estimate of sfermion mass difference in the sfermion basis. To confront the NS scenario with the experimental bounds on FCNC processes [16], we still have to evaluate sfermion mass matrices in a basis that diagonalizes fermion Yukawa matrices; specifically we are interested in the mixings $\left(\delta_{12}\right)_{\mathrm{LL}, \mathrm{RR}}$ between the first two families of left-handed or right-handed sfermions. In this respect, the NS scenario has the advantage that both fermion and sfermion mass matrices are known if the anomalous dimensions are specified. If the first two families have the same anomalous dimension $\gamma_{f 1}^{*}=\gamma_{f 2}^{*}$ at the SC fixed point, the Yukawa-diagonalizing angle will be of $\mathcal{O}(1)$, but at the same time $\Gamma_{\tilde{f} 1}=\Gamma_{\tilde{f} 2}$ guarantees the complete mass degeneracy of sfermions. On the other hand, if $\gamma_{f 1}^{*}>\gamma_{f 2}^{*}$ and $\Gamma_{\tilde{f} 1}$ $>\Gamma_{\tilde{f} 2}$, the sfermions have nondegeneracy estimated by $\left(\Gamma_{\tilde{f} 2}^{-1}-\Gamma_{\tilde{f} 1}^{-1}\right) \Delta_{\tilde{f}}$, but the diagonalizing angle is as small as $\left(M_{C} / M_{0}\right)^{\gamma_{1}^{*}-\gamma_{2}^{*}}$, which gives an additional suppression to FCNC processes. In this way, we expect that approximate alignment happens.

For squarks, the degeneracy factors are fairly small already in the sfermion basis. Moreover, if the diagonalizing angles are of the order of the Cabibbo angle, we have $\left(\delta_{12}^{d}\right)_{\mathrm{LL}, \mathrm{RR}} \sim 0.22 \times \Delta_{\tilde{f}} \approx 1 \times 10^{-3}$. Using the constraint from the $K^{0}-\bar{K}^{0}$ system, i.e.,

\footnotetext{
${ }^{2}$ In the MSSM case with GUT relation of the gaugino masses, we do not expect that the presence of the $\alpha_{3} M_{3}^{2}$ term will significantly change the previous estimation of $\Delta_{\tilde{L}}$ and $\Delta_{\tilde{e}}$, unless it is associated with a large group-theoretical factor.
} 


$$
\left(\delta_{12}^{d}\right)_{\mathrm{LL}, \mathrm{RR}}<1.2 \times 10^{-3} \times\left(\frac{m_{\tilde{d}}}{500 \mathrm{GeV}}\right),
$$

it is required that $m_{d} \approx 500 \mathrm{GeV}$, which corresponds to $M_{3}$ $\gtrsim 500 \mathrm{GeV}$ as well as $m_{\tilde{e}} \gtrsim 50 \mathrm{GeV}$. Thus FCNC constraints will easily be satisfied in the squark sector.

On the other hand, the degeneracy factors are not small enough in the slepton sector. Then lepton flavor-violating processes such as $\mu \rightarrow e \gamma$ decay constrain the slepton masses. Of course, such constraints depend on the texture of the lepton Yukawa matrix generated by the NS mechanism. To see this explicitly, let us concentrate on the first two families and denote by $\theta_{\mathrm{L}}$ and $\theta_{\mathrm{R}}$ the mixing angles for left- and right-handed leptons, respectively. First consider the case in which left-handed leptons receive the same anomalous dimension $\gamma_{L 1}=\gamma_{L 2}$ from the SC dynamics. Then the first 2 $\times 2$ lepton Yukawa matrix takes, up to $\mathcal{O}(1)$ factors, the lopsided form

$$
\begin{aligned}
y_{i j} & \approx y_{22}\left(\begin{array}{ll}
\varepsilon & 1 \\
\varepsilon & 1
\end{array}\right), \\
\varepsilon & =\left(\frac{M_{C}}{M_{0}}\right)^{\gamma_{e 1}^{*}-\gamma_{e 2}^{*}} \sim \frac{m_{e}}{m_{\mu}}=5 \times 10^{-3},
\end{aligned}
$$

where the lepton mass hierarchy originates from the anomalous dimensions of the right-handed leptons. In this case, the left-handed leptons have $\mathcal{O}(1)$ mixing and that will be favorable from the viewpoint of neutrino oscillation. Interestingly, the left-handed sleptons are completely degenerate since $\gamma_{L 1}^{*}=\gamma_{L 2}^{*}$ implies $\Gamma_{L 1}=\Gamma_{L 2}$. Moreover, the right-handed leptons have the mixing angle of $\theta_{\mathrm{R}}=\mathcal{O}(\varepsilon)$, which gives an additional suppression to $\left(\delta_{12}^{\ell}\right)_{\mathrm{RR}}$. Thus we will have no strong constraint.

If mixing angles are larger than $\mathcal{O}\left(10^{-3}\right)$, the constraint becomes significant. For example, if both the mixing angles for left- and right-handed sectors are of order

$$
\theta_{\mathrm{L}} \sim \theta_{\mathrm{R}} \sim \sqrt{\frac{m_{e}}{m_{\mu}}}=7 \times 10^{-2},
$$

we have $\left(\delta_{12}^{\ell}\right)_{\mathrm{LL}} \sim 7 \times 10^{-2} \Delta_{\tilde{f}} \approx 3 \times 10^{-3}$ and $\left(\delta_{12}^{\ell}\right)_{\mathrm{RR}} \sim 7$ $\times 10^{-2} \Delta_{\tilde{f}} \approx 7 \times 10^{-3}$, and it is required that $m_{\tilde{L}} \gtrsim 100 \mathrm{GeV}$ and $m_{\tilde{e}} \gtrsim 200 \mathrm{GeV}$ from the constraint

$$
\left(\delta_{12}^{\ell}\right)_{\mathrm{LL}, \mathrm{RR}}<2.0 \times 10^{-3} \times\left(\frac{m_{\tilde{\ell}}}{100 \mathrm{GeV}}\right)^{2} .
$$

The constraint on $\left(\delta_{12}^{\ell}\right)_{\mathrm{RR}}$ requires that the gluino and squarks are heavier than $2 \mathrm{TeV}$, whereas that on $\left(\delta_{12}^{\ell}\right)_{\mathrm{LL}}$ corresponds to gluino and squarks heavier than $400 \mathrm{GeV}$. In general, the larger mixing angle the right-handed lepton sector has, the heavier sleptons are required from $\mu \rightarrow e \gamma$ decay. For example, it is required that $m_{\tilde{e}} \gtrsim 300 \mathrm{GeV}$ if $\theta_{\mathrm{R}} \geq 0.2$.

We remark that there are additional constraints coming from $\left(\delta_{12}\right)_{\mathrm{LR}}$, which are related to SUSY-breaking trilinear couplings $h_{i j} \widetilde{\psi}_{i} \widetilde{\psi}_{j} H$. In the present SCFT framework, the soft scalar masses are well controlled by the SC dynamics, but the so-called $A$ terms $A_{i j} \equiv h_{i j} / y_{i j}$ are not; the SC dynamics does give a suppression factor to $h_{i j}$, which is the same as the suppression factor of the corresponding Yukawa coupling $y_{i j}$. Therefore, even if the constraints on $\left(\delta_{12}\right)_{\mathrm{LL}, \mathrm{RR}}$ are satisfied, we should still take care of additional constraints on $\left(\delta_{12}\right)_{\text {LR }}$ from FCNC processes [11]. We need another mechanism to suppress $A$ terms, or to realize the complete alignment ${ }^{3}$ of $A_{i j}$. At any rate, we expect that the constraints coming from $\left(\delta_{12}\right)_{\mathrm{LR}}$ will be less severe than those from $\left(\delta_{12}\right)_{\mathrm{LL}, \mathrm{RR}}$, and to examine the latter is the subject of the following sections.

\section{SU(5) GUT COUPLED TO SC SECTOR}

For $M_{C}>10^{16} \mathrm{GeV}$, we are naturally led to consider the embedding of the MSSM into SUSY GUTs. In this section, we study the Georgi-Glashow SU(5) model, which breaks down to the MSSM at the scale $M_{X}=2 \times 10^{16} \mathrm{GeV}$. We assume $M_{C} \geqslant M_{X}$, i.e., the SC sector decouples at a larger scale than the GUT breaking scale.

As in the MSSM case of Sec. II B, we expect that each sfermion mass converges on

$$
m_{\tilde{f i}}^{2}\left(M_{C}\right)=\frac{1}{\Gamma_{\tilde{f} i}} C_{f 5} \alpha_{5}\left(M_{C}\right) M_{5}^{2}\left(M_{C}\right),
$$

where $\alpha_{5}\left(M_{C}\right)$ and $M_{5}\left(M_{C}\right)$ are the SU(5) gauge coupling and the gaugino mass at the scale $M_{C}$. Representations of quarks and leptons under $\mathrm{SU}(5)_{\mathrm{GG}}$ are shown in the fifth column of Table I. The sixth column shows $C_{f 5}$ for each matter field. On the other hand, the radiative correction in the GUT regime between $M_{C}$ and $M_{X}$ is obtained as

$$
\Delta m_{\tilde{f i}}^{2}\left(M_{C} \rightarrow M_{X}\right)=\frac{C_{f 5}}{2 b_{5}} I_{5}\left(M_{X}, M_{C}\right) M_{5}^{2}\left(M_{C}\right),
$$

where $b_{5}$ is the beta-function coefficient of $\alpha_{5}$, and $I_{5}\left(M_{X}, M_{C}\right)$ is defined by

$$
I_{5}\left(M_{X}, M_{C}\right) \equiv 1-\frac{\alpha_{5}^{2}\left(M_{X}\right)}{\alpha_{5}^{2}\left(M_{C}\right)} .
$$

Then the sfermion mass degeneracy may be estimated by the use of

$$
\Delta_{\tilde{f}}=\frac{C_{f 5} \alpha_{5}\left(M_{C}\right) M_{5}^{2}\left(M_{C}\right)}{\Delta m_{\tilde{f i}}^{2}\left(M_{C} \rightarrow M_{X}\right)+\Delta m_{\tilde{f} i}^{2}\left(M_{X} \rightarrow M_{Z}\right)},
$$

where $\Delta m_{\tilde{f i}}^{2}\left(M_{X} \rightarrow M_{Z}\right)$ denotes the radiative correction in the MSSM regime, Eq. (7), with $M_{C}=M_{X}$. The value of $\Delta_{\tilde{f}}$

\footnotetext{
${ }^{3}$ One way to achieve this possibility was discussed in Ref. [17], where the Yukawa couplings $y_{i j}$ by themselves are suppossed to have infrared fixed points. In models with extra dimensions, it was also argued [18] that, thanks to power-law behavior due to KaluzaKlein modes, hierarchical Yukawa couplings can be obtained at the IR fixed points. In this case, however, it seems difficult to realize finite mixing angles.
} 


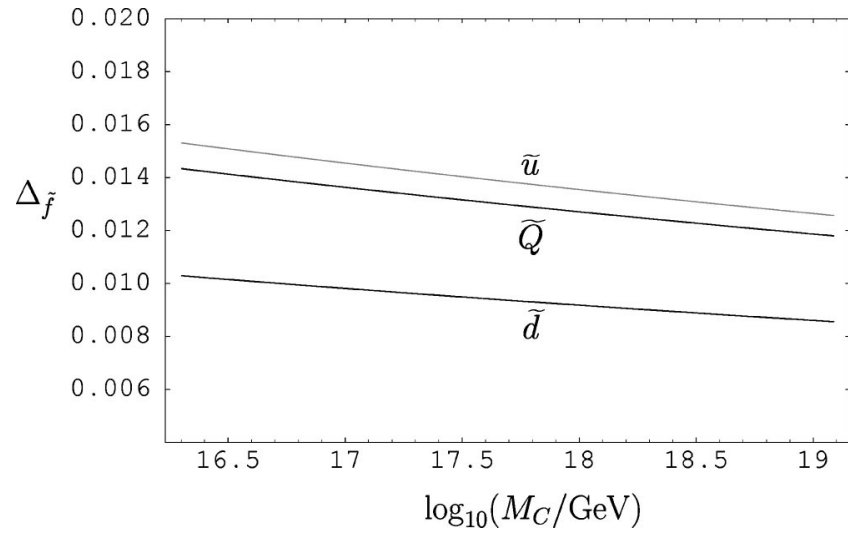

FIG. 1. $\Delta_{\tilde{Q}}, \Delta_{\tilde{u}}$, and $\Delta_{\tilde{d}}$ against $M_{C}$ in the SUSY SU(5) GUT.

depends on the flow of the gauge couplings $\alpha_{5}$ and $\alpha_{a=1,2,3}$, but not on the gaugino masses.

Figure 1 shows $\Delta_{\tilde{f}}$ for squarks and Fig. 2 for sleptons. For definiteness, we have taken $b_{5}=-9 / 2$ corresponding to the minimal SU(5), while different $b_{5}$ lead to similar results.

Compared with the MSSM case, Eqs. (11), (12), the mass spectrum at the weak scale is almost the same, but the degeneracy factors are not. First of all, the degeneracy factors for squarks and left-handed sleptons become slightly larger. Note also that $\Delta_{\tilde{Q}}$ and $\Delta_{\tilde{u}}$ are larger than $\Delta_{\tilde{d}}$, since $Q$ and $u$ belong to the $\mathbf{1 0}$ representation under SU(5) whereas $d$ belongs to the $\overline{\mathbf{5}}$, and the former has a larger convergent value than the latter. There is a small splitting between $\Delta_{\tilde{Q}}$ and $\Delta_{\tilde{u}}$ because $m_{\tilde{Q}}^{2}$ gets slightly larger radiative corrections in the MSSM regime from the $W$-ino mass $M_{2}$.

More importantly, the degeneracy for the right-handed slepton becomes drastically worse than before. The reason is very simple; the right-handed lepton is embedded into the $\mathbf{1 0}$ representation, which has a large Casimir coefficient, so that the convergent value (17) is enlarged. Consequently the FCNC constraint for $\left(\delta_{12}^{\ell}\right)_{\mathrm{RR}}$ cannot easily be satisfied, requiring heavier sleptons and/or a specific form of the lepton Yukawa matrix. It is rather surprising that this simple grouptheoretical fact precludes the SU(5) extension of the MSSM. At any rate, the embedding into SU(5) discussed here would remove one of the most interesting properties of the present

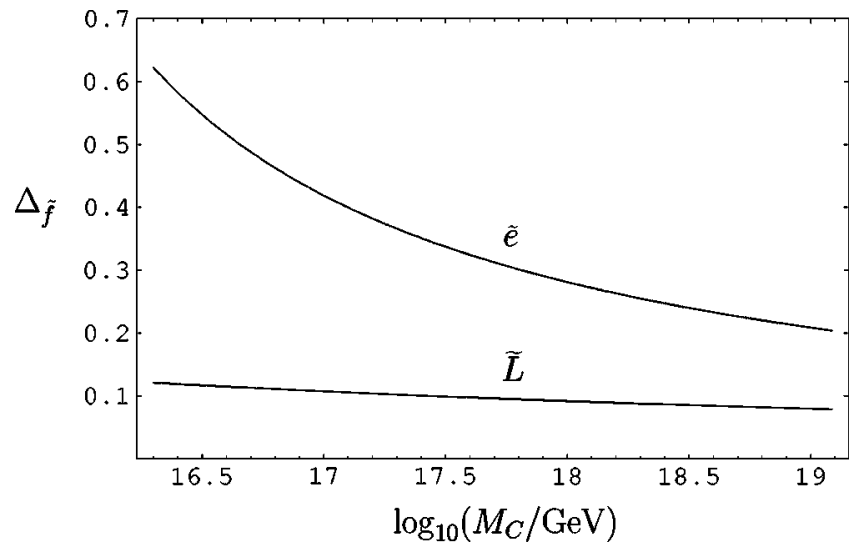

FIG. 2. $\Delta_{\tilde{e}}$ and $\Delta_{\tilde{L}}$ against $M_{C}$ in the SUSY SU(5) GUT.
TABLE II. The minimal flipped $\mathrm{SU}(5)_{F} \times \mathrm{U}(1)_{F}$ model. The last column shows $F \equiv \sqrt{40} \widetilde{X}$, where $\widetilde{X}$ is the $\mathrm{SO}(10)$-normalized $\mathrm{U}(1)_{F}$ charge of Eq. (21).

\begin{tabular}{cccc}
\hline \hline & $\begin{array}{c}\text { MSSM } \\
\text { content }\end{array}$ & $\begin{array}{c}\mathrm{SU}(5)_{F} \\
\text { representation }\end{array}$ & $\begin{array}{c}\mathrm{U}(1)_{F} \\
\text { charge }\end{array}$ \\
\hline$T_{i}$ & $d_{i}, Q_{i}, \nu_{R i}^{c}$ & $\mathbf{1 0}$ & +1 \\
$\bar{F}_{i}$ & $u_{i}, L_{i}$ & $\overline{\mathbf{5}}$ & -3 \\
$S_{i}$ & $e_{R i}^{c}$ & $\mathbf{1}$ & +5 \\
$h$ & $h_{d}^{c}, h_{d}$ & $\mathbf{5}$ & -2 \\
$\bar{h}$ & $h_{u}^{c}, h_{u}$ & $\overline{\mathbf{5}}$ & +2 \\
$H$ & - & $\overline{\mathbf{1 0}}$ & +1 \\
$\bar{H}$ & - & $\overline{\mathbf{1 0}}$ & -1 \\
\hline \hline
\end{tabular}

SCFT approach, that is, the approximate mass degeneracy of sfermions without specifying a mediation mechanism of SUSY breaking.

\section{FLIPPED SU(5) $\times$ U(1) COUPLED TO SC SECTOR}

We have observed that, within the framework of the ordinary $\mathrm{SU}(5) \mathrm{GUT}$, the degeneracy factor $\Delta_{\tilde{e}}$ is not small enough for the right-handed slepton because of its large Casimir coefficient. From this viewpoint, it is interesting to consider the flipped $\mathrm{SU}(5) \times \mathrm{U}(1)$ models [12], where the right-handed lepton is not embedded into a nontrivial SU(5) representation, but is an $\mathrm{SU}(5)$ singlet.

Let us first recall the basic feature of the minimal flipped $\mathrm{SU}(5)_{\mathrm{F}} \times \mathrm{U}(1)_{\mathrm{F}}$ model to fix our notation. The quantum numbers of matter and Higgs fields are shown in Table II, and the embedding of each family of matter fields is explicitly given by

$$
\begin{aligned}
& T=\left(\begin{array}{ccccc}
0 & d_{\mathrm{R} 3}^{c} & -d_{\mathrm{R} 2}^{c} & u_{1} & d_{1} \\
& 0 & d_{\mathrm{R} 1}^{c} & u_{2} & d_{2} \\
& & 0 & u_{3} & d_{3} \\
& & & 0 & \nu_{\mathrm{R}}^{c} \\
& & & & 0
\end{array}\right), \\
& \bar{F}=\left(\begin{array}{c}
u_{\mathrm{R} 1}^{c} \\
u_{\mathrm{R} 2}^{c} \\
u_{\mathrm{R} 3}^{c} \\
-e \\
\nu
\end{array}\right),
\end{aligned}
$$

and $S=e_{\mathrm{R}}^{c}$, where $\nu_{\mathrm{R}}^{c}$ is a right-handed neutrino. If the $\nu_{\mathrm{R}}^{c}$ components of the ten-dimensional Higgs fields $H$ and $\bar{H}$ develop the vacuum expectation values, the symmetry breaking to the MSSM can be achieved with a simple implementation of the missing partner mechanism for doublet-triplet splitting [19]. Through this symmetry breaking, the 24th generator $T_{\mathrm{F}}^{24} \equiv \sqrt{3 / 5} \tilde{Y}$ of $\mathrm{SU}(5)_{\mathrm{F}}$ and the $\mathrm{U}(1)_{\mathrm{F}}$ generator $F$ $\equiv \sqrt{40} \widetilde{X}$ are related to the hypercharge $Y$ as well as its orthogonal broken generator $X$. One way to describe this rela- 
tion is to embed the gauge group into $\mathrm{SO}(10)$ and to represent $\mathrm{U}(1)$ generators in the $\mathrm{SO}(10)$ basis. By picking its $\mathrm{SU}(4)_{\mathrm{PS}} \times \mathrm{SU}(2)_{\mathrm{L}} \times \mathrm{SU}(2)_{\mathrm{R}}$ subgroup, we have

$$
\begin{aligned}
\widetilde{X} & =\sqrt{\frac{3}{5}} T_{\mathrm{PS}}^{15}+\sqrt{\frac{2}{5}} T_{\mathrm{R}}^{3}, \\
\sqrt{\frac{3}{5}} \widetilde{Y} & =\sqrt{\frac{2}{5}} T_{\mathrm{PS}}^{15}-\sqrt{\frac{3}{5}} T_{\mathrm{R}}^{3} .
\end{aligned}
$$

Here $T_{\mathrm{R}}^{3}$ is the third generator of $\mathrm{SU}(2)_{\mathrm{R}}$ and $T_{\mathrm{PS}}^{15}=\sqrt{3 / 8}(B$ $-L)$ is the 15th generator of the Pati-Salam SU(4), which is proportional to the $B-L$ charge. Equation (21) is precisely the flipped version of the usual $\mathrm{SO}(10)$ relation

$$
\begin{aligned}
X & =\sqrt{\frac{3}{5}} T_{\mathrm{PS}}^{15}-\sqrt{\frac{2}{5}} T_{\mathrm{R}}^{3}, \\
\sqrt{\frac{3}{5}} Y & =\sqrt{\frac{2}{5}} T_{\mathrm{PS}}^{15}+\sqrt{\frac{3}{5}} T_{\mathrm{R}}^{3},
\end{aligned}
$$

where $X$ is nothing but the U(1) generator of $\mathrm{SO}(10) / \mathrm{SU}(5)_{\mathrm{GG}}$, often called $\mathrm{U}(1)_{\chi}$. Since these two sets of $\mathrm{U}(1)$ generators are orthogonally related to each other, we have

$$
\begin{aligned}
X & =\frac{1}{5} \widetilde{X}+\frac{\sqrt{24}}{5} T_{\mathrm{F}}^{24}, \\
\sqrt{\frac{3}{5}} Y & =\frac{\sqrt{24}}{5} \widetilde{X}-\frac{1}{5} T_{\mathrm{F}}^{24} .
\end{aligned}
$$

For notational simplicity, let $\alpha_{5}^{\prime}$ and $\alpha_{1}^{\prime}$ denote the gauge couplings of $\mathrm{SU}(5)_{\mathrm{F}} \times \mathrm{U}(1)_{\mathrm{F}}$. With the normalization of $\mathrm{U}(1)$ generators as above, the embedding into the $\mathrm{SO}(10)$ GUT would lead to $\alpha_{1}^{\prime}=\alpha_{5}^{\prime}$, although we do not assume such a further unification.

We assume that the SC sector decouples at the scale $M_{C}$, below which the model is exactly the same as the minimal flipped $\mathrm{SU}(5) \times \mathrm{U}(1)$ model. At the scale $M_{X}$ where $\mathrm{SU}(5) \times \mathrm{U}(1)$ breaks to the MSSM gauge group, the hypercharge gauge coupling $\alpha_{Y}=(3 / 5) \alpha_{1}$ is matched with $\mathrm{SU}(5) \times \mathrm{U}(1)$ gauge couplings $\alpha_{5}^{\prime}$ and $\alpha_{1}^{\prime}$ according to

$$
\frac{15}{\alpha_{Y}} \equiv \frac{25}{\alpha_{1}}=\frac{1}{\alpha_{5}^{\prime}}+\frac{24}{\alpha_{1}^{\prime}} .
$$

The measured value of the SM gauge couplings requires $\alpha_{1}^{\prime}\left(M_{X}\right) \approx \alpha_{5}^{\prime}\left(M_{X}\right) \approx(2 \pi \times 24.5)^{-1}$. The matching condition for the corresponding gaugino masses is given by

$$
\begin{aligned}
\frac{M_{1}}{\alpha_{1}} & =\frac{1}{25}\left(\frac{M_{5}^{\prime}}{\alpha_{5}^{\prime}}+\frac{24 M_{1}^{\prime}}{\alpha_{1}^{\prime}}\right) \\
& =\left(\frac{1+24 r}{25}\right) \frac{M_{5}^{\prime}}{\alpha_{5}^{\prime}}=\left(\frac{1+24 r}{25 r}\right) \frac{M_{1}^{\prime}}{\alpha_{1}^{\prime}} .
\end{aligned}
$$

Here we have introduced a RG-invariant quantity
TABLE III. The group-theoretical factors in the flipped SU(5) $\times \mathrm{U}(1)$ model. The last two columns show the "flipped hypercharge" $\widetilde{Y}$ and the $\mathrm{U}(1)_{\chi}$ charge $X$ in Eqs. (21), (22).

\begin{tabular}{cccccc}
\hline \hline$f$ & $\mathrm{SU}(5) \times \mathrm{U}(1)$ & $C_{f 5}^{\prime}$ & $C_{f 1}^{\prime}$ & $6 \tilde{Y}$ & $\sqrt{40} X$ \\
\hline$Q$ & $(\mathbf{1 0},+1)$ & $72 / 5$ & $1 / 10$ & +1 & +1 \\
$d_{R}^{c}$ & $(\mathbf{1 0},+1)$ & $72 / 5$ & $1 / 10$ & -4 & -3 \\
$\nu_{R}^{c}$ & $(\mathbf{1 0},+1)$ & $72 / 5$ & $1 / 10$ & +6 & +5 \\
$L$ & $(\overline{\mathbf{5}},-3)$ & $48 / 5$ & $9 / 10$ & -3 & -3 \\
$u_{R}^{c}$ & $(\overline{\mathbf{5}},-3)$ & $48 / 5$ & $9 / 10$ & +2 & +1 \\
$e_{R}^{c}$ & $(\mathbf{1},+5)$ & 0 & $5 / 2$ & 0 & +1 \\
\hline \hline
\end{tabular}

$$
r \equiv \frac{M_{1}^{\prime} / \alpha_{1}^{\prime}}{M_{5}^{\prime} / \alpha_{5}^{\prime}} \approx \frac{M_{1}^{\prime}\left(M_{X}\right)}{M_{5}^{\prime}\left(M_{X}\right)},
$$

which parametrizes the relative size of two independent gaugino masses of $\mathrm{SU}(5) \times \mathrm{U}(1)$. $\mathrm{SO}(10)$ gauge symmetry would require $r=+1$, but we treat $r$ as a free parameter. The gluino mass $M_{3}$ and the $W$-ino mass $M_{2}$ are matched as usual and are independent of $M_{1}^{\prime}$. However, the $B$-ino mass $M_{1}$ does depend on $r$, and the ratio $M_{1} / M_{3}$ is given by

$$
\frac{M_{1}}{M_{3}}=\left(\frac{1+24 r}{25}\right) \frac{\alpha_{1}}{\alpha_{3}}=\left(\frac{1+24 r}{25}\right) \frac{5 \alpha_{Y}}{3 \alpha_{3}} .
$$

We expect that the degeneracy factor for the right-handed sleptons also depends on $r$, since their masses will be dominantly governed by the $\mathrm{U}(1)$ gaugino masses.

It is straightforward to estimate the sfermion masses and confirm our expectation. The RGE for the soft scalar mass, which includes the leading term of the $\mathrm{SU}(5) \times \mathrm{U}(1)$ gauge loop, is

$$
\mu \frac{d m_{i}^{2}}{d \mu}=\mathcal{M}_{i j} m_{j}^{2}-\sum_{a=5,1} C_{i a}^{\prime} \alpha_{a}^{\prime} M_{a}^{\prime 2}
$$

where $C_{i a}^{\prime}$ denote the Casimir factors under $\mathrm{SU}(5) \times \mathrm{U}(1)$. Under the assumption that higher-order corrections to the second term are small, we may estimate the convergent value of each sfermion mass at $M_{C}$ and the degeneracy factor at the weak scale by

$$
\begin{aligned}
m_{\tilde{f} i}^{2}\left(M_{C}\right) & =\frac{1}{\Gamma_{\tilde{f} i}} \sum_{a=5,1} C_{f a}^{\prime} \alpha_{a}^{\prime}\left(M_{C}\right) M_{a}^{\prime 2}\left(M_{C}\right), \\
\Delta_{\tilde{f}} & =\frac{\sum_{a=5,1} C_{f a}^{\prime} \alpha_{a}^{\prime}\left(M_{C}\right) M_{a}^{\prime 2}\left(M_{C}\right)}{\Delta m_{\tilde{f} i}^{2}\left(M_{C} \rightarrow M_{X}\right)+\Delta m_{\tilde{f} i}^{2}\left(M_{X} \rightarrow M_{Z}\right)} .
\end{aligned}
$$

The group-theoretical factors are shown in Table III, and the radiative corrections in the $\mathrm{SU}(5) \times \mathrm{U}(1)$ regime can be calculated in a manner similar to Eq. (7) with $b_{5}^{\prime}=-5$ and $b_{1}^{\prime}$ $=15 / 2$. The results does not strongly depend on $M_{C}$, and we will take $M_{C}=M_{X}$ for definiteness. 


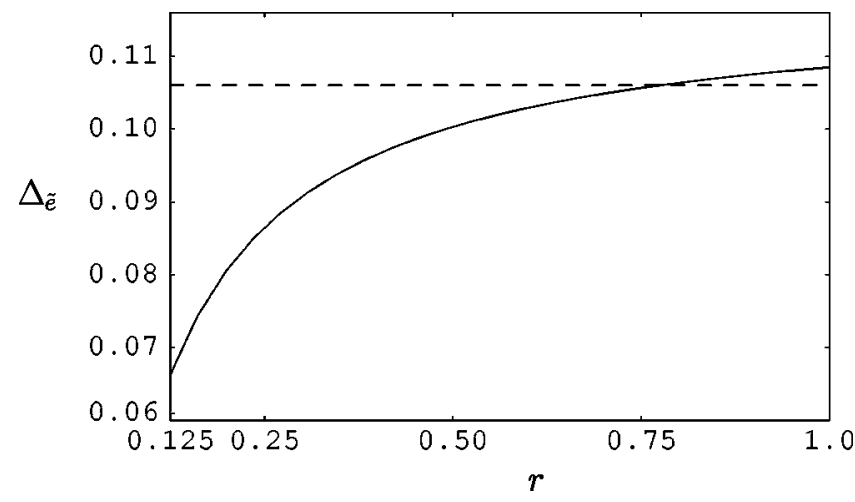

FIG. 3. The slepton degeneracy factor $\Delta_{\tilde{e}}$ against $r$ in the flipped $\mathrm{SU}(5) \times \mathrm{U}(1)$ model. The dashed line shows the value in the MSSM case.

We are especially interested in the expected degree of degeneracy $\Delta_{\tilde{e}}$ for the right-handed sleptons. The point is that the convergent value $m_{\tilde{e}}^{2}\left(M_{C}\right)$ is governed by the flipped $\mathrm{U}(1)$ gaugino mass $M_{1}^{\prime}$, whereas the radiative corrections $\Delta m_{\tilde{e}}^{2}$ are determined by the $B$-ino mass $M_{1}$, which contains as a component the $\mathrm{SU}(5)$ gaugino mass $M_{5}^{\prime}$ as well as $M_{1}^{\prime}$ according to the matching condition (25). Consequently, the degeneracy factor $\Delta_{\tilde{e}}$ behaves like

$$
\Delta_{\tilde{e}} \propto\left(\frac{M_{1}^{\prime}}{M_{1}}\right)^{2} \propto\left(\frac{25 r}{1+24 r}\right)^{2} .
$$

Numerical values for $\Delta_{\tilde{e}}$ are shown in Fig. 3 as a function of $r$, and we find explicitly

$$
\Delta_{\tilde{e}}= \begin{cases}6.6 \times 10^{-2} & \text { for } r=1 / 8, \\ 1.1 \times 10^{-1} & \text { for } r=1\end{cases}
$$

Compared with the MSSM case (11), a similar value of $\Delta_{\tilde{e}}$ is obtained in the $r=1$ case, as is expected from the fact that $r \approx 1$ corresponds to the usual GUT relation of gaugino masses. As we decrease $r$ keeping $M_{5}^{\prime}$ fixed, $M_{1}^{\prime}$ decreases linearly, whereas $M_{1}$ reaches a nonzero value, and thus $\Delta_{\tilde{e}}$ becomes small. In other words, the mass difference of $\widetilde{e}_{R}^{c}$ is determined by a small gaugino mass of the flipped $\mathrm{U}(1)$ while the $\mathrm{SU}(5)$ component of the B-ino mass effectively enhances the averaged slepton mass. In this way, we find that the degeneracy factor $\Delta_{\tilde{e}}$ is improved for $r \lessgtr 0.5$ compared with the MSSM case. For $|r| \gg 1$, the $B$-ino mass $M_{1}$ becomes proportional to $M_{1}^{\prime}$, and $\Delta_{\tilde{e}}$ approaches its maximal value 0.12 .

At first sight, the degeneracy factor $\Delta_{\tilde{e}}$ could be arbitrarily small if $M_{1}^{\prime} \ll M_{5}^{\prime}$. However, this is not true because our estimation based on the RGE (28) is no longer reliable for a hierarchically small value of $|r| \lesssim 0.1$. At the two-loop level, for instance, the RGE contains potentially dangeous terms like $\lambda_{e}^{2} \alpha_{5}^{\prime} M_{5}^{\prime 2}$ and $\alpha_{1}^{\prime} \alpha_{5}^{\prime} M_{1}^{\prime} M_{5}^{\prime}$, where $\lambda_{e}$ is the messenger coupling of the $\mathrm{SU}(5)$ singlet $e_{R}^{c}$. As mentioned in Sec. II B, the former term is absent if we assume that only SU(5)singlet fields couple to $e_{\mathrm{R}}^{c}$ through the messenger interaction. On the other hand, the latter term will give a substantial

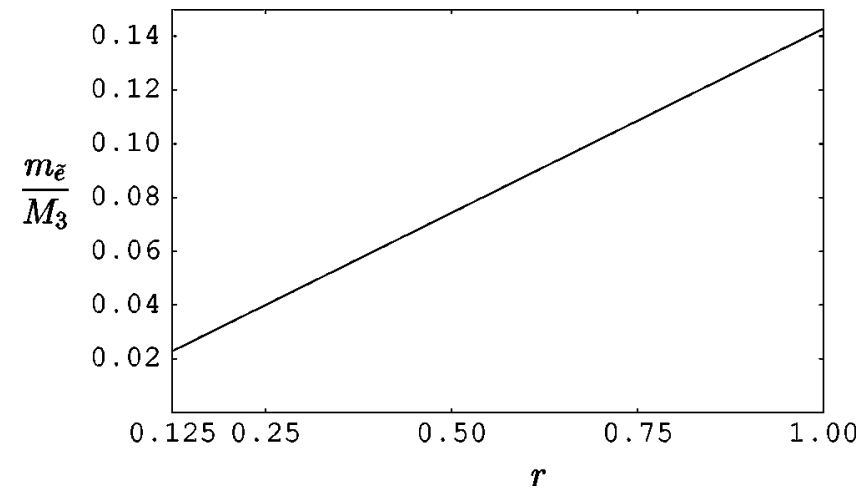

FIG. 4. $m_{\tilde{e}} / M_{3}$ against $r$ in the flipped $\mathrm{SU}(5) \times \mathrm{U}(1)$ model.

correction to $\Delta_{\tilde{e}}$ for such a small value of $|r|$. Even if the latter correction is properly taken into account, the ratio $M_{1} / M_{3}$ will be unacceptably small from the viewpoint of the naturalness.

The slepton mass at the weak scale is approximately

$$
m_{\tilde{e}}=0.93 M_{1}
$$

as long as the radiative correction $\Delta m_{\tilde{e}}^{2}$ dominates over the convergent value. Equation (27) then implies that the ratio $m_{\tilde{e}} / M_{3}$ decreases as we decrease $r$, as is shown in Fig. 4 .

For the other sfermions, the degeneracy factors and mass spectrum are determined dominantly by $\alpha_{a}$ and $M_{a}$ ( $a$ $=2,3$ ), and are almost independent of $r$ for a moderate range of the parameter $r \lesssim \mathcal{O}(2.5)$. Explicitly, we obtain, for $r$ $=1 / 8$,

$$
\begin{array}{ll}
\Delta_{\tilde{Q}}=1.5 \times 10^{-2}, & \Delta_{\tilde{L}}=1.3 \times 10^{-1}, \\
\Delta_{\tilde{d}}=1.6 \times 10^{-2}, & \Delta_{\tilde{u}}=1.1 \times 10^{-2},
\end{array}
$$

which are almost the same as in the SU(5) case, except that $\widetilde{u}$ and $\widetilde{d}$ are interchanged. The mass spectrum for squarks and left-handed sletpons is almost the same as in Eq. (12).

Note that $\Delta_{\tilde{L}}$ is larger than $\Delta_{\tilde{e}} \leqslant 0.12$ owing to the embedding into $\overline{\mathbf{5}}$. As discussed in Sec. IIC, the constraints from lepton flavor violations depend on the lepton mixing angles. For mixing angles (15), the constraints from $\left(\delta_{12}^{\ell}\right)_{\mathrm{LL}}$ require $m_{\tilde{\ell}} \gtrsim 200 \mathrm{GeV}$, which should be compared with $m_{\tilde{e}}$ $\geq 200 \mathrm{GeV}$ from $\left(\delta_{12}^{\ell}\right)_{\mathrm{RR}}$ in the MSSM with the same mixing angles. The former corresponds to $M_{3} \gtrsim 1 \mathrm{TeV}$, while the latter requires $M_{3} \gtrsim 2 \mathrm{TeV}$. Thus the flipped $\mathrm{SU}(5) \times \mathrm{U}(1)$ model, unlike the SU(5) models, is on the level of the MSSM.

\section{THRESHOLD EFFECTS}

Up to now, we have smoothly connected RG flows of soft scalar masses at $M_{X}$ as well as $M_{C}$. We reexamine this procedure and briefly discuss possible threshold effects of various sorts. 


\section{A. D-term contributions in flipped models}

When the rank of the gauge group is reduced, the gauge symmetry breaking induces a $D$-term contribution to the soft scalar mass squared, $\Delta_{D} m_{\tilde{f}}^{2}=q_{f}\langle D\rangle$, which is proportional to the charge of the broken $U(1)$ symmetry $[3,4]$. Here we consider the effects of such additional terms at $M_{X}$ induced by $\mathrm{SU}(5) \times \mathrm{U}(1)$ breaking. Of course, the charges under the broken symmetry are the same between different families, and those do not create nondegeneracy. However, such additional contributions change the overall magnitude of sfermion masses at the GUT threshold, and also change the mass spectrum and degeneracy factors at the weak scale.

The $D$-term contributions induced through the breaking of $\mathrm{SU}(5) \times \mathrm{U}(1)$ can be expressed in terms of the broken generators $F \equiv \sqrt{40} \widetilde{X}$ and $\widetilde{Y} \equiv \sqrt{5 / 3} T_{\mathrm{F}}^{24}$ in Eq. (21) as

$$
\Delta_{D} m_{\tilde{f}}^{2}=\frac{1}{5}\left(F_{f} \alpha_{1}^{\prime 2}+24 \tilde{Y}_{f} \alpha_{5}^{\prime 2}\right) m_{D}^{2}
$$

The magnitude of $m_{D}^{2}$ can be calculated when we explicitly fix the model and its breaking pattern. To be generic, however, let us take $m_{D}^{2}$ to be a free parameter. With the help of $\alpha_{5}^{\prime}\left(M_{X}\right) \approx \alpha_{1}^{\prime}\left(M_{X}\right)$, the above expression can be rewritten as

$$
\begin{aligned}
\Delta_{D} m_{\tilde{f}}^{2} & \approx \frac{\sqrt{40}}{5}\left(\widetilde{X}_{f}+\sqrt{24} T_{\mathrm{F} f}^{24}\right) \alpha_{1}^{\prime 2} m_{D}^{2} \\
& =\sqrt{40} X_{f}\left\langle D_{\chi}\right\rangle,
\end{aligned}
$$

where $X$ is the $\mathrm{U}(1)_{\chi}$ generator (23) in the $\mathrm{SO}(10)$ normalization, and $\left\langle D_{\chi}\right\rangle \equiv \alpha_{5}^{2} m_{D}^{2}$. Using the values of $\mathrm{U}(1)_{\chi}$ charges shown in Table III, we have

$$
\Delta_{D} m_{\tilde{L}}^{2}=-3\left\langle D_{\chi}\right\rangle, \quad \Delta_{D} m_{\tilde{e}}^{2}=\left\langle D_{\chi}\right\rangle,
$$

which give opposite effects on left- and right-handed slepton masses. Thus it is not possible to improve the degeneracy for both of them at the same time by the $D$-term contribution. ${ }^{4}$

Equation (33) indicates that the right-handed sleptons tend to be the LSPs. Therefore a positive contribution $\Delta_{D} m_{\tilde{e}}^{2}>0$ is favored to make them heavier, and this slightly improves the degeneracy for the right-handed sleptons. However, the degeneracy for left-handed sleptons becomes worse. For instance, we have $\Delta_{\tilde{L}}=2.1 \times 10^{-1}$ if we take $\left\langle D_{\chi}\right\rangle$ $=0.1 M_{5}^{\prime 2}\left(M_{X}\right)$.

\section{B. Note on SC threshold effects}

When the strongly coupled SC sector decouples around the scale $M_{C}$, there arises another type of threshold correction, which we refer to as SC threshold effects. We have little

\footnotetext{
${ }^{4} D$-term contributions through the breaking $E_{6} \rightarrow \mathrm{SO}(10)$ can be used [20] to increase the slepton masses, which are originally tachyonic in a scenario of anomaly-mediated SUSY breaking [21]. Such contributions might also be helpful here since the broken U(1) charge takes the same sign for all quarks and leptons.
}

to say about such effects; one could in principle evaluate SC threshold effects once the SC sector is specified, but it requires hard work in understanding the strong dynamics. Basically we do not expect that the Yukawa hierarchy generated by large anomalous dimensions will be modified so much by SC threshold effects, provided that the SC sector decouples quickly [5]. We have assumed that the same is true in our estimation of sfermion masses and degeneracy.

There is a special case in which SC threshold effects are reliably calculated. Consider for definiteness the MSSM coupled to the SC sector, whose decoupling is caused by invariant mass terms of the order $M_{C}$. Suppose also that all the soft SUSY-breaking parameters are given purely by anomaly mediation [21]. Even in this special case, one can evaluate as before the "convergent value" of the sfermion mass, which is actually the same as the anomaly-mediated one calculated by using beta functions of the MSSM coupled to the SC sector. On the other hand, as was argued in Ref. [22], the mass parameter $M_{C}$ should be extended to a background (nondynamical) superfield, and its $F$ term will affect the soft terms. One then expects that there will arise SC threshold effects such that, after the decoupling, sfermion masses are precisely the anomaly-mediated ones calculated in the MSSM framework. Thus the SC threshold effects are calculable in this case, although they are of no interest because of the tachyonic sleptons.

There is an interesting puzzle here. Originally we were interested in the SCFT approach because it can provide a degenerate sfermion spectrum no matter how the SUSY is mediated; if we consider RG flows of each sfermion mass corresponding to various initial conditions at the cutoff scale, all the flows converge on a certain value thanks to the SC dynamics. Such RG flows would contain a special flow corresponding to the anomaly-mediated spectrum. Now, suppose that SUSY breaking is not mediated purely by anomaly mediation. Nevertheless, the above convergence property of RG flows would imply that the sfermion spectrum at the decoupling scale, and thus the SC threshold effects, will be almost the same as the anomaly-mediated one. Specifically, the MSSM coupled to the SC sector would always lead to tachyonic sleptons no matter how the SUSY is initially mediated.

The resolution of this apparent puzzle is that the convergent value of each sfermion mass $m_{\tilde{f}}^{2}$ is independent of the initial conditions for $m_{\tilde{f}}^{2}$, but does depend on the MSSM gaugino masses. Therefore the sfermion mass spectrum can be different from the anomaly-mediated one, provided that the MSSM gaugino masses are different.

At any rate, a lesson is that SC threshold effects do affect sfermion masses, and it is interesting to calculate these SC threshold effects explicitly.

\section{CONCLUSION AND DISCUSSION}

We have studied sfermion mass degeneracy within the framework of GUTs coupled to SC sectors. In the SU(5) 
model, the degeneracy factor $\Delta_{\tilde{e}}$ for the right-handed sleptons becomes worse than in the MSSM. The reason is that the right-handed lepton $e_{\mathrm{R}}^{c}$ is embedded into the tendimensional representation and the convergent mass value is enlarged. Models with larger gauge groups like $\mathrm{SO}(10)$ and $E_{6}$ will have the same feature, as long as the SC sector decouples at a scale larger than the GUT breaking scale. One way to keep $e_{\mathrm{R}}^{c}$ a non-Abelian singlet is the flipped $\mathrm{SU}(5)_{\mathrm{F}}$ $\times \mathrm{U}(1)_{\mathrm{F}}$ model. In this model, the degeneracy of the righthanded sleptons can be improved if the $\mathrm{U}(1)_{\mathrm{F}}$ gaugino mass $M_{1}^{\prime}$ is smaller than the $\mathrm{SU}(5)_{\mathrm{F}}$ one; a small $M_{1}^{\prime}$ reduces the convergent value and the mass difference, while the $\mathrm{SU}(5)_{\mathrm{F}}$ component of the $B$-ino mass enhances the averaged slepton mass.

In this paper, we have estimated sfermion mass degeneracy without specifying the model for the SC sector. To this end, we have assumed that the convergent value of the sfermion masses at the decoupling scale is dominantly determined by the MSSM one-loop terms in the RGEs. This assumption is plausible for the $\mathrm{SU}(5)$ case, but is crucial especially for the right-handed sleptons in the MSSM and the flipped model. A clever model building will be required when the SC sector is vectorlike under the SM-sector gauge group. We have also assumed that our estimation is not substantially modified by SC threshold effects. We expect that the size of such effects will be at most comparable to the estimated convergent value of the sfermion masses. It is worth examining these points by explicit calculations.

Finally, let us speculate about possible extensions of the present work. We have neglected effects from the third family of quarks and leptons and their Yukawa couplings. In the quark sector, it is safe to neglect the third family, since they have only small mixings with the others in many realistic Yukawa matrices. However, the same is not true in the lepton sector. In particular, the flipped $\mathrm{SU}(5) \times \mathrm{U}(1)$ model requires that the tau neutrino Yukawa coupling is as large as the top Yukawa coupling. In general, the large mixings in the lepton sector induce a significant flavor violation $\delta_{12}^{\ell}$ through the radiative corrections above the mass scale $M_{\nu}$ of the right-handed neutrinos [23-26], which constrains the sfermion mass spectrum and/or requires a specific form of Yukawa matrices. From this viewpoint, an interesting possibility is that a proper coupling to the SC sector and subsequent IR convergence property can eliminate such flavor violation as well if $M_{C}$ is taken below $M_{\nu}=10^{13}-10^{15} \mathrm{GeV}$. We need further study concerning neutrino Yukawa couplings. ${ }^{5}$

In models with a product gauge group like SU(5)

\footnotetext{
${ }^{5} \mathrm{An}$ application of the SC idea to the neutrino as well as Higgs sectors was discussed in Ref. [27].
}

$\times \mathrm{U}(1)$, the degeneracy can be improved if the gaugino masses do not satisfy the usual GUT relation so that the convergent value of the sfermion mass is much suppressed compared with the radiative corrections in the MSSM regime. The $\mathrm{SU}(5) \times \mathrm{U}(3)_{\mathrm{H}}$ models [28] will have a similar property. We speculate that, even with a simple gauge group $\mathrm{SU}(5)$, such a suppression of the convergent value could be realized if one supposes that the SU(5) gauge multiplets live in extra-dimensional space-times. In this setup, the powerlaw evolution of the gauge coupling and gaugino mass [2931] makes them extremely small above $M_{X}$ (in such a way that $M_{5} / \alpha_{5}$ is still $\mathrm{RG}$ invariant [30]) provided that the gauge coupling is asymptotically free. Consequently, the degeneracy will be improved if $M_{C}$ is taken as the energy scale where $\alpha_{5}$ and $M_{5}$ are very small. To keep our RG calculations reliable in the SC regime, quarks and leptons as well as $\mathrm{SC}$-sector fields are supposed to live in four dimensions. Such a setup could also explain why our gauge coupling $\alpha_{5}$ is smaller than that in the SC sector, thanks to extradimensional volume suppression.

The Nelson-Strassler scenario, on which we have focused in this paper, assumes that the anomalous dimensions of quarks and leptons take flavor-dependent values at the SC fixed point. The difference between $\gamma_{i}^{*}$ and $\gamma_{j}^{*}$ leads to hierarchical Yukawa matrices, but at the same time the difference between $\Gamma_{i}$ and $\Gamma_{j}$ leads to a distinctive nondegeneracy of sfermion masses. In the "Yukawa hierarchy transfer" scenario [32], the SC dynamics is supposed to be flavor blind at the fixed point $\gamma_{i}^{*}=\gamma_{j}^{*}$ and $\Gamma_{i}=\Gamma_{j}$, ensuring complete degeneracy of sfermion masses. The hierarchy among Yukawa couplings $y_{i j}$ is derived by assuming an inverse hierarchy in the messenger couplings $\lambda_{\text {irs }}$ at the cutoff scale and by transferring the initial hierarchy through the SC dynamics. Moreover, the assumed hierarchy can be derived, e.g., through the FN mechanism in the SC sector without spoiling the sfermion degeneracy by family-dependent $D$-term contributions. This new scenario provides an alternative way of realizing the degenerate sfermion spectrum and hierarchical Yukawa matrices at the same time.

\section{ACKNOWLEDGMENTS}

The authors would like to thank J. Kubo, N. Maekawa, J. Sato, D. Suematsu, M. Tanimoto, and T. Yanagida for useful discussions. We would like to express our thanks to the organizers of the Summer Institute, Yamanashi, where a part of the work was done. This work is supported in part by a Grant-in-Aid for Scientific Research from Ministry of Education, Science, Sports and Culture of Japan (No. 03242). The work of T.N. is supported in part by the Japan Society for the Promotion of Science under the Predoctoral Research Program.
[1] C. D. Froggatt and H. B. Nielsen, Nucl. Phys. B147, 277 (1979).

[2] L. E. Ibáñez and G. G. Ross, Phys. Lett. B 332, 100 (1994).
[3] M. Drees, Phys. Lett. B 181, 279 (1986); J. S. Hagelin and S. Kelley, Nucl. Phys. B342, 95 (1990); Y. Kawamura, H. Murayama, and M. Yamaguchi, Phys. Lett. B 324, 94 (1994); 
Phys. Rev. D 51, 1337 (1995).

[4] H. Nakano, hep-th/9404033; Prog. Theor. Phys. Suppl. 123, 387 (1996); Y. Kawamura and T. Kobayashi, Phys. Lett. B 375, 141 (1996); 388, 867(E) (1996); Phys. Rev. D 56, 3844 (1997); E. Dudas, S. Pokorski, and C. A. Savoy, Phys. Lett. B 369, 255 (1996); E. Dudas, C. Grojean, S. Pokorski, and C. A. Savoy, Nucl. Phys. B481, 85 (1996).

[5] A. E. Nelson and M. J. Strassler, J. High Energy Phys. 09, 030 (2000).

[6] T. Banks and A. Zaks, Nucl. Phys. B196, 189 (1982).

[7] N. Seiberg, Nucl. Phys. B435, 129 (1995).

[8] A. Karch, T. Kobayashi, J. Kubo, and G. Zoupanos, Phys. Lett. B 441, 235 (1998).

[9] M. A. Luty and R. Rattazzi, J. High Energy Phys. 11, 001 (1999).

[10] T. Kobayashi and H. Terao, Phys. Rev. D 64, 075003 (2001).

[11] A. E. Nelson and M. J. Strassler, J. High Energy Phys. 07, 021 (2002).

[12] S. M. Barr, Phys. Lett. 112B, 219 (1982); J. P. Derendinger, J. E. Kim, and D. V. Nanopoulos, ibid. 139B, 170 (1984); I. Antoniadis, J. Ellis, J. S. Hagelin, and D. V. Nanopoulos, Phys. Lett. B 194, 231 (1987).

[13] M. A. Luty and R. Sundrum, Phys. Rev. D 65, 066004 (2002); hep-th/0111231.

[14] H. Terao, hep-ph/0112021.

[15] Y. Yamada, Phys. Rev. D 50, 3537 (1994); J. Hisano and M. Shifman, ibid. 56, 5475 (1997); I. Jack and D. R. T. Jones, Phys. Lett. B 415, 383 (1997); I. Jack, D. R. T. Jones, and A. Pickering, ibid. 426, 73 (1998); 432, 114 (1998); L. V. Avdeev, D. I. Kazakov, and I. N. Kondrashuk, Nucl. Phys. B510, 289 (1998); T. Kobayashi, J. Kubo, and G. Zoupanos, Phys. Lett. B 427, 291 (1998); N. Arkani-Hamed, G. F. Giudice, M. A. Luty, and R. Rattazzi, Phys. Rev. D 58, 115005 (1998); D. I. Kazakov and V. N. Velizhanin, Phys. Lett. B 485, 393 (2000).

[16] F. Gabbiani, E. Gabrielli, A. Masiero, and L. Silverstrini, Nucl. Phys. B477, 321 (1996). See also for a review, e.g., M. Misiak,
S. Pokorski, and J. Rosiek, hep-ph/9703442; J. L. Feng, hep-ph/0101122, and references therein.

[17] T. Kobayashi and K. Yoshioka, Phys. Rev. D 62, 115003 (2000).

[18] M. Bando, T. Kobayashi, T. Noguchi, and K. Yoshioka, Phys. Lett. B 480, 187 (2000); Phys. Rev. D 63, 113017 (2001).

[19] A. Masiero, D. V. Nanopoulos, K. Tamvakis, and T. Yanagida, Phys. Lett. 115B, 380 (1982).

[20] M. Carena, K. Huitu, and T. Kobayashi, Nucl. Phys. B592, 164 (2000).

[21] L. Randall and R. Sundrum, Nucl. Phys. B557, 79 (1999); G. F. Giudice, M. A. Luty, H. Murayama, and R. Rattazzi, J. High Energy Phys. 12, 27 (1998).

[22] A. Pomarol and R. Rattazzi, J. High Energy Phys. 05, 013 (1999).

[23] F. Borzumati and A. Masiero, Phys. Rev. Lett. 57, 961 (1986).

[24] J. Hisano, T. Moroi, K. Tobe, M. Yamaguchi, and T. Yanagida, Phys. Lett. B 357, 579 (1995); J. Hisano, T. Moroi, K. Tobe, and T. Yanagida, Phys. Rev. D 53, 2442 (1996).

[25] J. Sato, K. Tobe, and T. Yanagida, Phys. Lett. B 498, 189 (2001); J. Sato and K. Tobe, Phys. Rev. D 63, 116010 (2001).

[26] A. Kageyama, S. Kaneko, N. Shimoyama, and M. Tanimoto, Phys. Lett. B 527, 206 (2002).

[27] J. Kubo and D. Suematsu, Phys. Rev. D 64, 115014 (2001).

[28] T. Yanagida, Phys. Lett. B 344, 211 (1995); T. Hotta, K.-I. Izawa, and T. Yanagida, Phys. Rev. D 53, 3913 (1996); 54, 6970 (1996); K.-I. Izawa and T. Yanagida, Prog. Theor. Phys. 97, 913 (1997); Y. Imamura, T. Watari, and T. Yanagida, Phys. Rev. D 64, 065023 (2001).

[29] K. Dienes, E. Dudas, and T. Gherghetta, Phys. Lett. B 436, 311 (1998); Nucl. Phys. B537, 47 (1999).

[30] T. Kobayashi, J. Kubo, M. Mondragon, and G. Zoupanos, Nucl. Phys. B550, 99 (1999).

[31] J. Kubo, H. Terao, and G. Zoupanos, Nucl. Phys. B574, 495 (2000).

[32] T. Kobayashi, H. Nakano, and H. Terao, Phys. Rev. D 65, 015006 (2002); (in preparation). 